\title{
Investigation of memory-enhancing botanical mixture and their isolated compounds for inhibition of amyloid- $\beta$ and tau aggregation
}

\author{
Seungyeop Baek ${ }^{1,2,3,4}$ (D), Sohui Park ${ }^{2,3,5}$, Jisu Shin' ${ }^{2,3}$, Jun-Seok Lee ${ }^{4}$, Hye Yun Kim ${ }^{3,5^{*}} \mathbb{D}$, Gyoonhee Han 1,2,3* \\ and YoungSoo $\mathrm{Kim}^{2,3,5^{*}}$ (1)
}

\begin{abstract}
Alzheimer's disease (AD) is a neurodegenerative disease characterized by the abnormal assembly of amyloid- $\beta$ (A $\beta$ ) and tau aggregates in the brain. When $A \beta$ and tau proteins misfold, progressive brain cell death, synaptic loss, atrophy, and cognitive decline are observed. Here, we report that the memory-enhancing botanical natural product mixture, $\mathrm{HX} 106 \mathrm{~N}$, efficiently inhibits formation of A $\beta$ oligomers and fibrils and aggregation of tau. HX106N is a botanical mixture extract of Dimocarpus longan, Liriope platyphylla, Salvia miltiorrhiza and Gastrodia elata. In previous clinical studies, HX106N showed increased working memory performances of individuals of subjective memory complaints. However, the drug mechanism and responsible ingredients of HX106N has been unclear yet. In this study, we expanded the investigation of the drug mode of action to the single chemical level by identifying four active components of $\mathrm{HX} 106 \mathrm{~N}$, among 14 isolated molecules, with significant inhibitory function against $A \beta$ aggregation. We found that salvianolic acids A, B, E and rosmarinic acid, isolated from the botanical mixture, have potency to inhibit the protein misfolding.
\end{abstract}

Keywords: Dimocarpus longan, Liriope platyphylla, Salvia miltiorrhiza, Gastrodia elata, Alzheimer's disease

\section{Introduction}

Alzheimer's disease (AD) is the most common type of dementia and misfolding of amyloid- $\beta(A \beta)$ and tau proteins in the brain is the key characteristics of the disorder. The pathogenesis behind the cognitive decline of AD patients is the significant atrophy of brain regions responsible for learning and memory due to the loss of neuronal cells and synapses by toxic aggregates of $A \beta$ and tau $[1,2]$. Studies suggested that amyloid plaque is associated with the onset of $\mathrm{AD}$ and tau tangle is correlated with the disease progression [3, 4]. Thus, the abnormal aggregation of these two proteins has been the prominent targets for $\mathrm{AD}$

\footnotetext{
*Correspondence: hyeyunkim@yonsei.ac.kr; gyoonhee@yonsei.ac.kr; y.kim@yonsei.ac.kr

${ }^{3}$ Yonsei Institute of Pharmaceutical Sciences, Yonsei University, 85 Songdogwahak-ro, Yeonsu-gu, Incheon 21983, Republic of Korea Full list of author information is available at the end of the article
}

therapy [5]. Early drug discoveries to regulate $A \beta$ and tau targeted the sequential proteolysis of the amyloid precursor protein and kinases inducing hyperphosphorylation, respectively $[6,7]$. Recent therapeutic approaches mostly aim the direct regulation of $A \beta$ or tau aggregation instead of altering upstream mechanisms in the disease cascade to bypass side-effects $[8,9]$. Successful clinical trials of the antibody drug candidate aducanumab, of Biogen, to remove $A \beta$ plaques from patient brains is a representative example of such approaches [10]. However, the two key protein targets are mostly not handled at the same time, despite the important pathogenic roles of both $A \beta$ or tau aggregation [8]. An additional issue to consider is the effect of these disease-modifying drugs on cognitive decline while protein misfolding is regulated. So far, none has clearly demonstrated recovery or termination of cognitive decline in clinical trials yet, and, thus, the 
correlation between memory and protein misfolding is still on debates.

In this study, we focused on a previously reported botanical mixture, HX106N, which ameliorated subjective memory complaints in clinical studies and Alzheimer-like memory impairments in mice [11, 12]. HX106N is a water-soluble fraction extracted from a mixture of four plants selected for their positive effects on brains: Dimocarpus longan, Liriope platyphylla, Salvia miltiorrhiza, and Gastrodia elata for their drug-like activities on increasing brain-derived neurotrophic factor levels, inducing neurite outgrowth [13], anti-oxidation/antiinflammation [14], and neuroprotection [15], respectively. In a clinical trial with 75 individuals who report subjective memory complaints, seven-week long administration of HX106N resulted in an increase in working memory performance [11]. HX106N was studied using an $\mathrm{AD}$ mouse model induced by the injection of $\mathrm{A} \beta(25-$ 35 ) and effectively alleviated memory impairment by reducing oxidative stress [12]. Albeit expected memory amelioration was observed in both preclinical and clinical studies, the exact mechanism of HX106N to help the symptom relief has not been clearly investigated yet. Thus, here, we examined the possible anti-amyloid and anti-tau effects of HX106N in in vitro assays to understand its mode of action. We tested the dose-dependent activity of HX106N against insoluble $\beta$-sheet-rich aggregate formation of $\mathrm{A} \beta$ and tau in thioflavin $\mathrm{T}$ (ThT) assays. We also monitored inhibition of oligomeric $A \beta$ formation by dose-dependent treatment of HX106N to monomeric $A \beta$ in gel electrophoresis and dot blots. Lastly, we assessed inhibition effects on the protein misfolding of 14 single chemical components of HX106N in ThT assays to discover the responsible ingredients of the anti-dementia effects of HX106N.

\section{Materials and methods \\ Reagents}

Thioflavin T (ThT, \#T3516), dimethyl sulfoxide (DMSO, \#D8418), tween 20 (\#P6416) were purchased from SigmaAldrich. We used skim milk from Difco ${ }^{\mathrm{TM}}(\# 232100)$ and phosphate-buffered saline from Gibco (PBS, pH 7.4, \#11010-023). All reagents and devices for the electrophoresis were purchased from Bio-Rad: mini-PROTEAN TGX gel (\#456-1095), tris/glycine/SDS buffer (\#1610772). ECL solution was purchased from Thermo scientific (\#34580). PlusOne protein silver staining kit was purchased from GE healthcare (\#17-1150-01). Antibodies for immunoblotting were anti-amyloidogenic protein oligomer A11 (\#AHB0052, Invitrogen) and HRP-linked anti-rabbit IgG (\#6401-05, Biovision). Deionized water was produced by Milli-Q plus ultrapure water system from Millipore.

\section{Preparation of $\mathrm{HX} 106 \mathrm{~N}$}

HX106N was extracted and prepared from four plants, Dimocarpus longan, Liriope platyphylla, Salvia miltiorrhiza, and Castrodia elata, as previously reported. Briefly, all plants were dried, grinded, and boiled in distilled water to extract the soluble fraction. The extract was filtered and lyophilized [12].

\section{Peptide synthesis}

We synthesized A $\beta$ on TentaGel ${ }^{\circledR}$ PHB resin (\#R28013, Rapp Polymere) via fluorenylthetyloxycarbonyl (Fmoc) solid phase peptide synthesis, using a peptide synthesizer (Liberty Blue, CEM). The reagents used are as follows: $N, N$-diisopropylcarbodiimide (DIC, \#D0254, TCI) as a coupling reagent; ethyl 2-cyano-2-(hydroxyimino) acetate (Oxyma, OxymaPure, \#S001-D, CEM corporation) as a coupling base; and $20 \%$ piperidine (\#A12442, Alfa Aesar) as a Fmoc deprotection reagent. Crude $\mathrm{A} \beta(1-42)$ was purified using RP-HPLC with a diphenyl column, as previously described [16].

\section{ThT fluorescence assay}

Synthetic A $\beta(1-42)$ peptides were dissolved in DMSO as $10 \mathrm{mM}$ stock. Stocks were then diluted 200 -fold in deionized water to make $A \beta(1-42)$ solutions $(50 \mu \mathrm{M}$, $1 \%$ DMSO in DW). For the inhibition assay, $A \beta(1-42)$ peptides were added to different concentrations of HX106N (10, 50, 100, 250, 500, and $1000 \mu \mathrm{g} / \mathrm{mL})$ and incubated for 3 days $(72 \mathrm{~h})$ at $37^{\circ} \mathrm{C}$. Also, 14 selected components of HX106N $(500 \mu \mathrm{g} / \mathrm{mL}, 1 \%$ DMSO in DW) was incubated with $50 \mu \mathrm{M} \mathrm{A} \beta(1-42)$ for 3 days at $37{ }^{\circ} \mathrm{C}$. For the dissociation assay, three-day incubated $50 \mu \mathrm{M} \mathrm{A} \beta(1-42)$ peptides were added to different concentrations of HX106N (31, 62 and $125 \mu \mathrm{g} / \mathrm{mL}$ ) and further incubated for 2 days $(48 \mathrm{~h})$ at $37^{\circ} \mathrm{C}$. At the end of incubation, $\mathrm{A} \beta$ oligomerization was analyzed with ThT assay. ThT ( $5 \mu \mathrm{M}$ in $50 \mathrm{mM}$ glycine buffer, $\mathrm{pH} 8.9)$ was added to black Corning 96-well plate with aggregated $\mathrm{A} \beta$ samples.

The K18 fragment (125 amino acids, $0.5 \mathrm{mg} / \mathrm{mL}$ ) cloned from full-length human tau was used for tau aggregation as previously reported [17]. The tau K18 fragment $(35 \mu \mathrm{M})$ in PBS was incubated with $0.1 \mathrm{mg} /$ $\mathrm{mL}$ heparin (Sigma-Aldrich) and dithiothreitol (DTT; $100 \mu \mathrm{M})$ (Sigma-Aldrich) at $37^{\circ} \mathrm{C}$ for 5 days $(120 \mathrm{~h})$. HX106N was added to the aggregation mixtures prior to a five-day incubation period. At the end of incubation, K18 oligomerization was analyzed with ThT assay.

Fluorescence of ThT bound to $A \beta$ or K18 was measured at $450 \mathrm{~nm}$ (excitation) and $485 \mathrm{~nm}$ (emission) using Multimode Plate Readers (TECAN Infinite M200 PRO). 


\section{SDS-PAGE with PICUP}

$\mathrm{A} \beta$ samples were irradiated for $3 \mathrm{~s}$ in $1.5 \mathrm{~mL}$ tube, three times, to cross-link $A \beta$ with tris(bipyridine)ruthenium(II) chloride (\#224758, Sigma-Aldrich) and ammonium persulfate (\#A3678, Sigma-Aldrich). Cross-linked A $\beta$ samples were applied on $15 \%$ tris-tricine gels. After gel electrophoresis, peptide bands on gels were stained and visualized by silver-staining [18].

\section{Dot blot}

Incubated $A \beta(1-42)$ samples were applied to nitrocellulose membrane, then, it was blocked with $5 \%$ skim milk in tris-buffered saline with tween 20 (TBST) at room temperature for $1 \mathrm{~h}$ and washed with TBST for three times. Probe antibody used was Oligomer A11 antibody (1:1000) with $2.5 \%$ skim milk in TBST solution, incubated overnight at $4{ }^{\circ} \mathrm{C}$. After incubation and washing three times at TBST, it was probed with anti-rabbit IgG, HRP-linked antibody $(1: 10,000)$ with TBST solution for $1 \mathrm{~h}$ at room temperature. The dot blots were developed with Fusion Solo (Vilber) for 3 to $6 \mathrm{~min}$ and scanned, analyzed with Fusion software.

\section{UHPLC-MS analysis}

Chromatographic analysis was performed on an Agilent 1290 UHPLC equipped with DAD (Agilent Technologies, Inc.). An Agilent ZORBAX SB-C18 Rapid Resolution HD column $(2.1 \times 100 \mathrm{~mm}, 1.8 \mu \mathrm{m})$ was applied for chromatographic separations. The mobile phase consisted of water containing $0.1 \%(v / v)$ formic acid (A) and acetonitrile (B). The UHPLC system was eluted under the following conditions: $0-3 \mathrm{~min}, 8 \% \mathrm{~B} ; 3-4 \mathrm{~min}, 8-10 \%$ B; 4-6 min, $10-16 \% \mathrm{~B}$; 6-15 min, $16 \% \mathrm{~B}$; $15-18 \mathrm{~min}$, $16-18 \% \mathrm{~B} ; 18-23 \mathrm{~min}, 18-18.2 \% \mathrm{~B} ; 23-25 \mathrm{~min}, 18.2-$ $18.5 \% \mathrm{~B} ; 25-29 \mathrm{~min}, 18.5-25 \% \mathrm{~B} ; 29-31 \mathrm{~min}, 25-50 \%$ B; 31-33 $\mathrm{min}, 50-70 \% \mathrm{~B}$; 33-34 $\mathrm{min}, 70-100 \% \mathrm{~B}$; 34-35 $\mathrm{min}, 100 \% \mathrm{~B}$. The flow rate was set at $0.5 \mathrm{~mL} / \mathrm{min}$ and the injection volume was $2 \mu \mathrm{L}$. The column temperature was kept at $30^{\circ} \mathrm{C}$.

The MS analysis was performed on an Agilent 6530 Q-TOF-MS equipped with electrospray ionization (ESI) interface (Agilent Technologies, Inc.) operating in negative ion mode, using the following operation parameters: drying gas $\left(\mathrm{N}_{2}\right)$ flow rate, $1.0 \mathrm{~L} / \mathrm{min}$; drying gas temperature, $350{ }^{\circ} \mathrm{C}$; nebulizer pressure, $35 \mathrm{psi}$; sheath gas temperature, $350{ }^{\circ} \mathrm{C}$; sheath gas flow, $11 \mathrm{~L} / \mathrm{min}$; capillary voltage, $3500 \mathrm{~V}$; fragmentor voltage, $100 \mathrm{~V}$.

\section{Statistical analysis}

All graphs were obtained with GraphPad Prism 7.0, and all statistical analyses were conducted with one-way
ANOVA followed by Bonferroni's post hoc comparisons $\left({ }^{*} P<0.05,{ }^{* *} P<0.01,{ }^{* * *} P<0.001\right)$. The error bars represent the SEM.

\section{Results and discussion}

Both $A \beta$ plaques and tau tangles share a similar $\beta$-sheetrich tertiary structure, which can be detected by $\beta$-sheetintercalating chemicals and ThT is a commonly used chemical $\beta$-sheet indicator to monitor and quantify the progressive formation of insoluble $A \beta$ and tau aggregates in vitro [19]. Upon binding to $\beta$-sheets, ThT shows increased fluorescence intensity with a red shift. To examine the effect of $\mathrm{HX} 106 \mathrm{~N}$ on $\mathrm{A} \beta$ fibril formation, we incubated monomeric $\mathrm{A} \beta(1-42)(50 \mu \mathrm{M})$ for 3 days at $37{ }^{\circ} \mathrm{C}$ with $\mathrm{HX} 106 \mathrm{~N}$ in a dose-dependent manner, 0,10 , $50,100,250,500$, and $1000 \mu \mathrm{g} / \mathrm{mL}$. Following the completion of the co-incubation, ThT solution was added to each sample and the degree of $\mathrm{A} \beta$ fibril formation, indicated by altered fluorescence of ThT, was immediately monitored (Fig. 1a). To compare data, we normalized the fluorescence intensity of the incubated $A \beta$ sample without HX106N addition to $100 \%$ and compared relative amounts of A $\beta$ fibrils in each sample. Samples with concentrations higher than $100 \mu \mathrm{g} / \mathrm{mL}$ significantly prevented the formation of $A \beta$ fibril. At $1000 \mu \mathrm{g} / \mathrm{mL}$, the highest concentration of $\mathrm{HX} 106 \mathrm{~N}$ in the assay, more than $60 \%$ of fibrils were reduced $\left(\mathrm{IC}_{50}=544.3 \mu \mathrm{g} / \mathrm{mL}\right)$. This result implies that $\mathrm{HX} 106 \mathrm{~N}$ has a dose-dependent inhibitory function against $\mathrm{A} \beta$ fibrillogenesis. To examine the effect of HX106N on tau aggregation, we performed ThT assay with the recombinant K18 tau fragment, which is a critical binding domain in tau aggregation [17]. K18 was incubated with heparin as a cofactor for 5 days at $37{ }^{\circ} \mathrm{C}$ with $\mathrm{HX} 106 \mathrm{~N}$ in a dose-dependent manner, $0,10,100,1000$, and $10,000 \mu \mathrm{g} / \mathrm{mL}$. When the co-incubation is completed, we added ThT solution to each sample and observed altered fluorescence of ThT. As a result, we observed a significant reduction of ThT signals when $10 \mathrm{mg} / \mathrm{mL}$ of $\mathrm{HX} 106 \mathrm{~N}$ was treated with K18 tau fragment, indicating that HX106N inhibits tau aggregation (Fig. 1b). When identical concentrations of HX106N were tested as used in the A $\beta$ aggregation inhibition tests, a dose-dependent inhibitory effect was not observed and, thus, we decided to focus on the anti-A $\beta$ effects of HX106N for further experiments.

As ThT assays are limited to detect $\beta$-sheet-rich fibrils only, we expanded the assessment to visualize $A \beta$ oligomers and monomers. Instead of typical western blots using antibodies, measurements of monomers, oligomers, and fibrils on electrophoresis gel analyses often utilize methods to cross-link aggregates by PICUP chemistry and to dye proteins by silver staining [18]. To examine the effect of $\mathrm{HX} 106 \mathrm{~N}$ on the formation of $\mathrm{A} \beta$ oligomers and fibrils, 

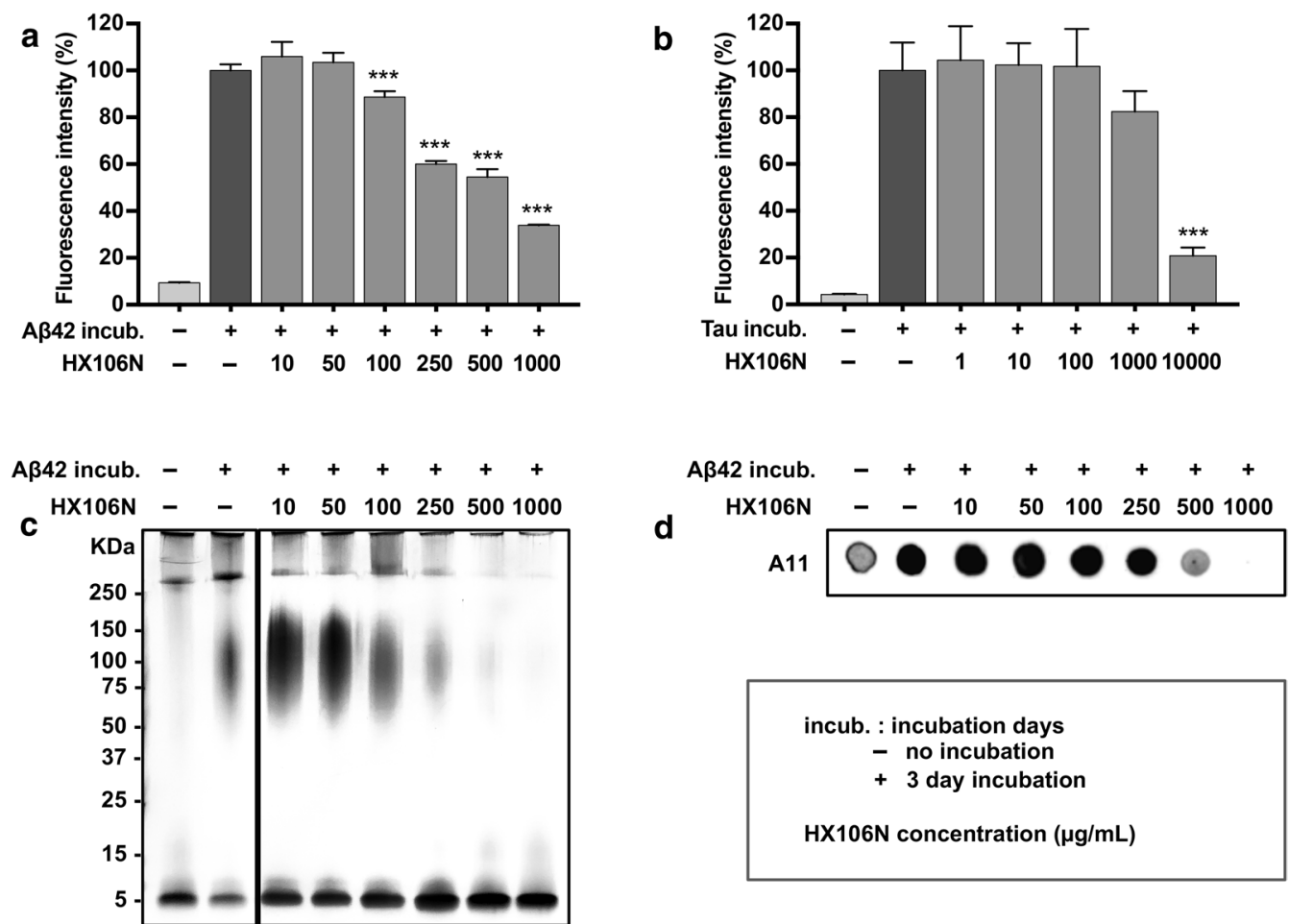

HX106N concentration $(\mu \mathrm{g} / \mathrm{mL})$

Fig. $1 \mathrm{HX} 106 \mathrm{~N}$ inhibits $A \beta$ and tau aggregation by blocking the transition of monomers into oligomers. a ThT assay for the inhibition of $A \beta(1-42)$ aggregation. Six concentrations of $\mathrm{HX} 106 \mathrm{~N}$ were incubated with equal amounts of monomeric $A \beta(1-42)$. Fluorescence intensity was normalized to $A \beta$ aggregates (100\%, 3-day). All data are representative results of at least three independent experiments. $\mathbf{b}$ ThT assay for the inhibition of tau aggregation. Five concentrations of $\mathrm{HX} 106 \mathrm{~N}$ were incubated with equal amounts of tau. Fluorescence intensity was normalized to tau aggregates (100\%, 5-day). All data are representative results of at least three independent experiments. $\mathbf{c}$ Visualization of A 3 peptide distribution by SDS-PAGE analysis with PICUP chemistry. $\mathbf{d}$ Dot blot analysis of protein oligomer (anti-amyloidogenic protein oligomer A11) in incubated samples of A $\beta$ and HX106N

we incubated monomeric $\mathrm{A} \beta(1-42)(50 \mu \mathrm{M})$ for 3 days at $37{ }^{\circ} \mathrm{C}$ with $\mathrm{HX} 106 \mathrm{~N}$ in a dose-dependent manner, 0,10 , 50, 100, 250, 500, and $1000 \mu \mathrm{g} / \mathrm{mL}$. After cross-linking, each sample was loaded on SDS gels for electrophoresis and then silver-stained (Fig. 1c). We analyzed the bands around $5 \mathrm{kDa}$ as monomers, above $250 \mathrm{kDa}$ as fibrils, and those between as oligomers. Consistent with the result of the ThT assay, incubated $A \beta$ samples with increasing concentrations of HX106N decreased fibrils correspondingly. In addition, the formation of oligomers between approximately 37 and $150 \mathrm{kDa}$ was effectively inhibited at higher concentrations, $250-1000 \mu \mathrm{g} / \mathrm{mL}$. This indicates that HX106N significantly inhibits the formation of fibrils and furthermore, reduces the total amount of toxic oligomers. Notably, the sample treated with the highest concentration of HX106N displayed a thicker monomer band in comparison to the non-aggregated $\mathrm{A} \beta$ sample. This implies that HX106N either interferes with the cross-linking of $A \beta$ peptides during the PICUP process or reverses $A \beta$ aggregation. In fact, HX106N showed a tendency to dissociate pre-aggregated $A \beta$ fibrils in a disaggregation ThT assay (Additional file 1: Figure S1c). In addition, we considered that the color of the HX106N stock could interfere with the cross-linking chemistry, although HX106N did not have a noticeable color at experimental concentrations. In order to observe the changes in the level of $A \beta$ oligomers without the influence of color, dot blot assay was performed using the anti-amyloidogenic protein oligomer (A11) antibody on the incubated samples of HX106N and $A \beta(1-42)$. We confirmed that high concentrations of HX106N are able to reduce toxic $A \beta$ oligomer formation (Fig. 1d). Taken together, $\mathrm{HX} 106 \mathrm{~N}$ binds to monomeric $\mathrm{A} \beta$ and prevents its transformation into oligomers and fibrils, blocking $A \beta$ aggregation at an early pathological stage.

As an extract from four botanical sources, HX106N was expected to be a mixture of various chemical compounds. To determine the components of HX106N, we performed UHPLC-MS analyses and identified 14 major compounds (Fig. 2a), which were all originated from Salvia miltiorrhiza and Gastrodia elata. Each molecule was tested in ThT assays to find components responsible for 
a

\begin{tabular}{ccc}
\hline No. & Identification & Plant source \\
\hline $\mathbf{1}$ & Citric acid & Gastrodia elata \\
$\mathbf{2}$ & Gastrodin & Gastrodia elata \\
$\mathbf{3}$ & Protocatechuic aldehyde & Salvia miltiorrhiza \\
$\mathbf{4}$ & Parishin A & Gastrodia elata \\
$\mathbf{5}$ & Salvianolic acid A & Salvia miltiorrhiza \\
$\mathbf{6}$ & Salvianolic acid B & Salvia miltiorrhiza \\
$\mathbf{7}$ & Rosmarinic acid & Salvia miltiorrhiza \\
$\mathbf{8}$ & Tanshinol & Salvia miltiorrhiza \\
$\mathbf{9}$ & Parishin E & Gastrodia elata \\
$\mathbf{1 0}$ & Parishin B & Gastrodia elata \\
$\mathbf{1 1}$ & Parishin C & Gastrodia elata \\
$\mathbf{1 2}$ & Salvianolic acid C & Salvia miltiorrhiza \\
$\mathbf{1 3}$ & Salvianolic acid D & Salvia miltiorrhiza \\
$\mathbf{1 4}$ & Salvianolic acid E & Salvia miltiorrhiza \\
\hline & &
\end{tabular}

\section{C}

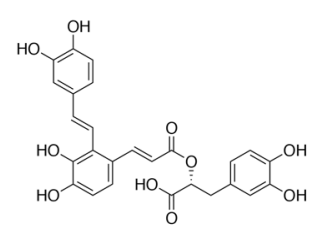

Salvianolic acid A (5)

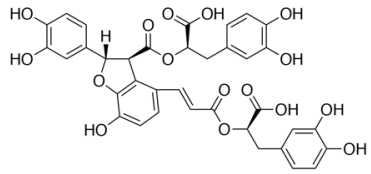

Salvianolic acid B (6)

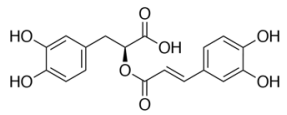

Rosmarinic acid (7)

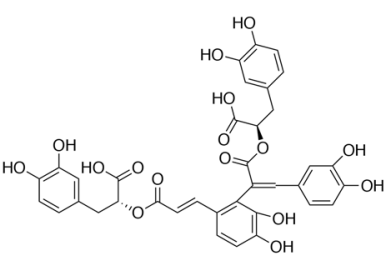

Salvianolic acid E (14)

Fig. 2 ThT assay for the inhibition of A 3 aggregation by selected components of $H X 106 \mathrm{~N}$. a The chemical components of HX106N used in the A $\beta$ aggregation inhibition test. $\mathbf{b}$ ThT assay for the inhibition of $A \beta(1-42)$ aggregation. $500 \mu \mathrm{g} / \mathrm{mL}$ of selected compounds were incubated with equal amount of monomeric $A \beta(1-42)$. Fluorescence intensity was normalized to $A \beta$ aggregates (100\%, 3-day). All data are representative results of at least three independent experiments. c Chemical structures of salvianolic acids A, B, E, and rosmarinic acid

the inhibition ability of $\mathrm{HX} 106 \mathrm{~N}$ against $\mathrm{A} \beta$ aggregation (Fig. 2b). The 14 chemicals were incubated with $50 \mu \mathrm{M}$ $\mathrm{A} \beta(1-42)$ for 3 days at $37{ }^{\circ} \mathrm{C}$, and the degree of $\mathrm{A} \beta$ fibril formation was measured by ThT assays. Among tested molecules, salvianolic acid A, B, E, and rosmarinic acid showed significant inhibition activity (Fig. 2c). In addition, citric acid and salvianolic acid D also showed a relatively weak but substantial effect to inhibit $A \beta$ aggregation (Fig. 2b, component numbers 1 and 13).

Collectively, different concentrations of HX106N were tested in ThT fluorescence assay to investigate its inhibition ability in $A \beta(1-42)$ aggregation. Less fluorescence signal was observed in HX106N-treated samples, indicating reduced fibril formation. In SDSPAGE with PICUP and silver staining, we observed lower fibrillization with increasing concentration of HX106N. Dot blot assay was done to confirm the effect of HX106N treatment in $A \beta$ oligomer formation, and we observed less oligomers in samples with high concentrations of HX106N. In addition, we identified the single molecules of HX106N and found that HX106N includes several molecules with inhibitory function against $A \beta(1-42)$ aggregation. In our study, salvianolic acid $A, B, E$, and rosmarinic acid showed significant inhibition effect by blocking the formation of mature $\beta$-sheet structure formation. Salvianolic acid A and $B$ were previously reported to inhibit $A \beta(1-40)$ and $A \beta(1-42)$ aggregation [20-22], while inhibition of oligomer and fibril formation was observed with salvianolic acid A and rosmarinic acid [23, 24]. By doing a ThT assay with each of these compounds, we observed a direct inhibition effect against $A \beta$ fibril formation. As the four active compounds discovered in our study are from Salvia miltiorrhiza, A $\beta$ aggregation inhibition can be expected upon treatment of Salvia miltiorrhiza alone. However, $\mathrm{AD}$ has various factors which worsen its severity other than $A \beta$ aggregation, such as oxidative stress and inflammation [25]. Given that citric acid and gastrodin from Gastrodia elata and Dimocarpus longan are anti-oxidative and anti-inflammatory components, administration of $\mathrm{HX} 106 \mathrm{~N}$ as a mixture might induce synergistic effect [26]. In addition, a previous study demonstrates that Liriope platyphylla helps active absorption of nutrients by promoting vascular flow [27]. The promising results from previous preclinical and clinical trials with HX106N administration provide evidence that compounds extracted from the four plants have complementary effect to enhance the general health of an $A D$ patient by not only targeting $A \beta$ accumulation, but also other symptoms. 
Current $\mathrm{AD}$ therapeutic development has its major focus on reducing $A \beta$ and tau levels in the brain [8]. In this study, we demonstrate that $\mathrm{HX} 106 \mathrm{~N}$, a water-soluble botanical formulation, inhibits both $A \beta$ and tau aggregation. The ability of HX106N to prevent the formation of mature $\beta$-sheet structures and toxic $A \beta$ oligomers indicates its potential in reducing $A \beta$-induced toxicity, which may have been the reason behind its positive results in clinical studies.

The long delay between $A \beta$ accumulation and the onset of symptoms makes it extremely difficult to diagnose and treat $\mathrm{AD}$ patients at an early stage, who already have high amyloid content without significant cognitive decline [24]. With these difficulties, the lack of disease-modifying drug increases the interest in developing preventive means that can be administered casually by individuals without clinical symptoms. WHO reported that approximately $80 \%$ of the world's population depend on plant derivative medicines for their healthcare, which are the earliest medicine as its application has a long history back from ancient traditional medicine systems [28]. Long use of natural products in traditional medicine can be considered as a clinical trial that proves their low toxicity and side effects, making its application in today's therapeutics easier. In fact, medicines today are still inspired by the vast information accumulated during the practice of traditional phytomedicine in Asia. This increases the advantage of HX106N being a natural extract that inhibits $A \beta$ and tau aggregation, given that it can be potentially used as a nutraceutical ingredient for individuals with a risk of developing $\mathrm{AD}$. Although the effect of long-term administration of HX106N needs to be further studied, HX106N has a promising potential as a plant-based nutraceutical considering the shorter drug development period of natural product than chemical or biological drug. Therefore, our study suggests that investigating the clinical application of natural products can be a powerful approach for AD prevention.

\section{Supplementary information}

Supplementary information accompanies this paper at https://doi. org/10.1186/s13765-020-00507-z.

Additional file 1: Figure S1. HX106N inhibits and reverses A $\beta$ aggregation by blocking the transformation of monomers into oligomers. a Full-length SDS-PAGE gel corresponding to Fig. 1b. b Full-length dot blot membrane corresponding to Fig. 1c. c ThT assay for the disaggregation of $A \beta(1-42)$ aggregates. Three concentrations of $H X 106 \mathrm{~N}$ were incubated with equal amounts of $A B(1-42)$. Fluorescence intensity was normalized to $A \beta$ aggregates (100\%, 3-day). All data are representative results of at least three independent experiments.

\section{Abbreviations}

AD: Alzheimer's disease; Aß: Amyloid- $\beta$; DIC: N,N-Diisopropylcarbodiimide; DMSO: Dimethyl sulfoxide; DTT: Dithiothreitol; ESI: Electrospray ionization;
Fmoc: Fluorenylthetyloxycarbonyl; Oxyma: Ethyl 2-cyano-2-(hydroxyimino) acetate; PBS: Phosphate-buffered saline; TBST: Tris-buffered saline with tween 20: ThT: Thioflavin T.

\section{Acknowledgements}

This work was supported by Korea Health Industry Development Institute (HI18C0836), National research Foundation of Korea (NRF2018R1D1A1B07048857) and ViroMed.

\section{Authors' contributions}

SYB performed the experiment, data analysis and interpretation, and wrote the final manuscript. SP analyzed data and drafted the manuscript. JS performed the experiment, data analysis. JSK and HYK contributed materials and editorials. GH and YSK supervised the project and revised the final manuscript. All authors read and approved the final manuscript.

\section{Availability of data and materials}

Datasets used and/or analyzed during the current study that are not included in the manuscript are available from the corresponding author on reasonable request.

\section{Competing interests}

All authors declare that they have no conflict of interests.

\section{Author details}

${ }^{1}$ Department of Biotechnology, Yonsei University, 50 Yonsei-ro, Seodaemun-gu, Seoul 03722, Republic of Korea. ${ }^{2}$ Department of Pharmacy, Yonsei University, 85 Songdogwahak-ro, Yeonsu-gu, Incheon 21983, Republic of Korea. ${ }^{3}$ Yonsei Institute of Pharmaceutical Sciences, Yonsei University, 85 Songdogwahak-ro, Yeonsu-gu, Incheon 21983, Republic of Korea. ${ }^{4}$ Molecular Recognition Research Center, Korea Institute of Science and Technology, 5. Hwarang-ro 14-gil, Seongbuk-gu, Seoul 02792, Republic of Korea. ${ }^{5}$ Integrated Science and Engineering Division (ISED), Yonsei University, 85 Songdogwahak-ro, Yeonsu-gu, Incheon 21983, Republic of Korea.

Received: 1 March 2020 Accepted: 26 April 2020

Published online: 06 May 2020

\section{References}

1. Crouch PJ, Harding SM, White AR, Camakaris J, Bush Al, Masters CL (2008) Mechanisms of Abeta mediated neurodegeneration in Alzheimer's disease. Int J Biochem Cell Biol 40(2):181-198. https://doi.org/10.1016/j. biocel.2007.07.013

2. Iqbal K, Liu F, Gong CX, Grundke-labal I (2010) Tau in Alzheimer disease and related tauopathies. Curr Alzheimer Res 7(8):656-664. https://doi. org/10.2174/156720510793611592

3. Murphy MP, LeVine H 3rd (2010) Alzheimer's disease and the amyloidbeta peptide. J Alzheimers Dis 19(1):311-323. https://doi.org/10.3233/ jad-2010-1221

4. Ballatore C, Lee VMY, Trojanowski JQ (2007) Tau-mediated neurodegeneration in Alzheimer's disease and related disorders. Nat Rev Neurosci 8(9):663-672. https://doi.org/10.1038/nrn2194

5. Bloom GS (2014) Amyloid- $\beta$ and Tau: the trigger and bullet in Alzheimer disease pathogenesis. JAMA Neurology 71(4):505-508. https://doi. org/10.1001/jamaneurol.2013.5847

6. Kumar D, Ganeshpurkar A, Kumar D, Modi G, Gupta SK, Singh SK (2018) Secretase inhibitors for the treatment of Alzheimer's disease: long road ahead. Eur J Med Chem 148:436-452. https://doi.org/10.1016/j.ejmec h.2018.02.035

7. Brunden KR, Trojanowski JQ, Lee VMY (2009) Advances in tau-focused drug discovery for Alzheimer's disease and related tauopathies. Nat Rev Drug Discov 8(10):783-793. https://doi.org/10.1038/nrd2959

8. Cummings J, Lee G, Ritter A, Sabbagh M, Zhong K (2019) Alzheimer's disease drug development pipeline: 2019. Alzheimers Dement (N Y) 5:272-293. https://doi.org/10.1016/j.trci.2019.05.008

9. Hung S-Y, Fu W-M (2017) Drug candidates in clinical trials for Alzheimer's disease. J Biomed Sci 24(1):47. https://doi.org/10.1186/s1292 9-017-0355-7 
10. Castrillo-Viguera C, Haeberlein SB, VonRosenstiel P, Chen T, O'Gorman J, Rajagovindan R et al (2019) Interim analyses of fixed-dose and titration cohorts from PRIME: a randomized, double-blind, placebocontrolled phase $1 \mathrm{~b}$ study of aducanumab (S9.006). Neurology 92(15 Supplement):S9.006

11. Kwon OLS, Ban S, Im JJ, Lee DS, Lee EH, Kim J, Lim SM, Lee SG, Kang I (2015) Effects of the combination herbal extract on working memory and white matter integrity in healthy individuals with subjective memory complaints: a randomized, double-blind, placebo-controlled clinical trial. Korean J Biol Psychiatry 22(2):63-77

12. Lee DS, Choi J, Kim SH, Kim S (2014) Ameliorating effects of HX106N, a water-soluble botanical formulation, on A 325 -35-induced memory impairment and oxidative stress in mice. Biol Pharm Bull 37(6):954-960. https://doi.org/10.1248/bpb.b13-00906

13. Hur J, Lee P, Kim J, Kim AJ, Kim H, Kim SY (2004) Induction of nerve growth factor by butanol fraction of liriope platyphylla in C6 and primary astrocyte cells. Biol Pharm Bull 27(8):1257-1260. https://doi.org/10.1248/ bpb.27.1257

14. Chien M-Y, Chuang C-H, Chern C-M, Liou K-T, Liu D-Z, Hou Y-C et al (2016) Salvianolic acid A alleviates ischemic brain injury through the inhibition of inflammation and apoptosis and the promotion of neurogenesis in mice. Free Radical Biol Med 99:508-519. https://doi.org/10.1016/j.freer adbiomed.2016.09.006

15. Huang N-K, Chern Y, Fang J-M, Lin C-I, Chen W-P, Lin Y-L (2007) Neuroprotective principles from gastrodia elata. 70(4):571-574. https://doi. org/10.1021/np0605182

16. Lee $S, \operatorname{Kim} Y$ (2015) Anti-amyloidogenic approach to access amyloid- $\beta(1-42)$ in fmoc solid-phase synthsis. Bull Korean Chem Soc 36(8):2147-2149. https://doi.org/10.1002/bkcs.10391

17. Haque MM, Kim D, Yu YH, Lim S, Kim DJ, Chang YT et al (2014) Inhibition of tau aggregation by a rosamine derivative that blocks tau intermolecular disulfide cross-linking. Amyloid 21(3):185-190. https://doi. org/10.3109/13506129.2014.929103

18. Bitan G (2006) Structural study of metastable amyloidogenic protein oligomers by photo-induced cross-linking of unmodified proteins. Methods Enzymol 413:217-236. https://doi.org/10.1016/s0076-6879(06)13012-8

19. Wolfe LS, Calabrese MF, Nath A, Blaho DV, Miranker AD, Xiong Y (2010) Protein-induced photophysical changes to the amyloid indicator dye thioflavin T. Proc Natl Acad Sci USA 107(39):16863-16868. https://doi. org/10.1073/pnas.1002867107
20. Durairajan SSK, Chirasani VR, Shetty SG, lyaswamy A, Malampati S, Song J et al (2017) Decrease in the generation of amyloid-beta due to salvianolic acid B by modulating BACE1 activity. Curr Alzheimer Res 14(11):12291237. https://doi.org/10.2174/1567205014666170417103003

21. Tang Y, Huang D, Zhang MH, Zhang WS, Tang YX, Shi ZX et al (2016) Salvianolic acid B inhibits Abeta generation by modulating BACE1 activity in SH-SY5Y-APPsw cells. Nutrients. https://doi.org/10.3390/nu8060333

22. Yu T, Paudel P, Seong SH, Kim JA, Jung HA, Choi JS (2018) Computational insights into beta-site amyloid precursor protein enzyme 1 (BACE1) inhibition by tanshinones and salvianolic acids from salvia miltiorrhiza via molecular docking simulations. Comput Biol Chem 74:273-285. https:// doi.org/10.1016/j.compbiolchem.2018.04.008

23. Hamaguchi T, Ono K, Murase A, Yamada M (2009) Phenolic compounds prevent Alzheimer's pathology through different effects on the amyloidbeta aggregation pathway. Am J Pathol 175(6):2557-2565. https://doi. org/10.2353/ajpath.2009.090417

24. Jackson HM, Soto I, Graham LC, Carter GW, Howell GR (2013) Clustering of transcriptional profiles identifies changes to insulin signaling as an early event in a mouse model of Alzheimer's disease. Bmc Genomics. https:// doi.org/10.1186/1471-2164-14-831

25. Agostinho P, Cunha RA, Oliveira C (2010) Neuroinflammation, oxidative stress and the pathogenesis of Alzheimer's disease. Curr Pharm Des 16(25):2766-2778. https://doi.org/10.2174/138161210793176572

26. Abdel-Salam OME, Youness ER, Mohammed NA, Morsy SMY, Omara EA, Sleem AA (2014) Citric acid effects on brain and liver oxidative stress in lipopolysaccharide-treated mice. J Med Food 17(5):588-598. https://doi. org/10.1089/jmf.2013.0065

27. Lee Y-J, Koh E-K, Kim J-E, Go J, Song S-H, Seong J-E et al (2015) Beneficial effects of ethanol extracts of Red Liriope platyphylla on vascular dysfunction in the aorta of spontaneously hypertensive rats. Lab Anim Res 31(1):13. https://doi.org/10.5625/lar.2015.31.1.13

28. Dar RA, Shahnawaz M, Rasool S, Qazi PH (2017) Natural product medicines: a literature update. Int J Phytopharm 6(6):340-342. https://doi. org/10.3390/metabo2020303

\section{Publisher's Note}

Springer Nature remains neutral with regard to jurisdictional claims in published maps and institutional affiliations.

\section{Submit your manuscript to a SpringerOpen ${ }^{\circ}$ journal and benefit from:}

- Convenient online submission

- Rigorous peer review

- Open access: articles freely available online

- High visibility within the field

- Retaining the copyright to your article

Submit your next manuscript at $\boldsymbol{\nabla}$ springeropen.com 